\title{
PERTURBACIONES DE LA INTELIGENCIA, SU EVALUACION Y SU TRATAMIENTO EN LA PARALISIS CEREBRAL INFANTIL
}

\author{
Dra. RAQUEL RIQUELME
}

Servicio de Neuropsiquiatria. Hospital "Roberto del Río". Santaga.

El niño con Parálisis Cerebral, como ya hemos visto, puede presentar grados muy diversos de compromiso motor, en igual forma la inteligencia puede estar lesionada en mayor o menor cuantía. Sin embargo, es preciso aclarar que no siempre hay una correlación estricta entre el compromiso psíquico y el neurológico. Muchas veces podremos encontrarnos ante un nin̄o que no obstante sus intensas alteraciones motoras conserva la inteligencia intacta, y en el extremo opuesto podemos hallar al niño que con un daño neurológico poco apreciable o moderado tiene una inteligencia muy disminuída. En éstos, como en todos los niños, existe desde luego un patrimonio intelectual hereditario, terreno sobre el que se marcará el daño cerberal; pero siendo el cerebro del nin̄o como una página en blanco, su lesión causará mayores trastornos que en el adulto cuya vida psíquica está ya formada. Además, si recordamos que el cerebro funciona como un todo, en que el trabajo de cada parte está intimamente vinculado a las demás, nos explicaremos más claramente el tipo de trastornos funcionales a que se ven expuestos.

El grupo que nos ocupa representa un tipo de daño cerebral que se exterioriza en forma grosera a través de un compromiso motor. Intentaremos ahora enfocar su repercusión en la esfera de la inteligencia y funciones psíquicas vecinas (o afines), aclarando que muchos de sus rasgos no les son exclusivos, sino que forman parte de todos los tipos de dañados cerebrales. Sin embargo, como estamos reunidos para tratar del niño con Parálisis Cerebral se enfocará en adelante la inteligencia y sus problemas desde este punto de vista.

Tal vez el defecto de mayor importancia, y, en parte, el configurador de otros, sea el de la percepción, que puede darse en niños de inteligencia normal o subnormal. En efecto, él condiciona en gran parte las dificultades de aprendizaje de los niños Paralíticos Cerebrales. Se ha dicho que la percepción es el proceso mental que le otorga un sentido y significación particular a una sensación dada y por consiguiente actúa como preliminar del pensamiento. Es decir, los sentidos recogen las sensaciones formándose al comienzo percepciones confusas y parcialmente organizadas que luego se relacionan a los objetos y acontecimientos circundantes, llegándose finalmente a la formación de conceptos y abstracciones sobre los objetos.

Todo niño normal explora el mundo que le rodea, formándose una experiencia sensorial a través de la cual aprende lo que es tamaño, forma, distancía, profundidad, etc. Es aquí donde se manifiestan las dificultades perceptivas del paralitico cerebral, ya que aunque pueden ver los objetos no logran apreciar las distancias que los separan o la que existe entre el objeto y ellos mismos. A menudo la falla no es tan grosera, pero se evidencia en su dificultad de distinguir en un dibujo la figura del fondo, de manera que al solicitarles una explicación sobre lo visualizado pueden describirnos el fondo en lugar de la figura.

No entienden tampoco un concepto claro de la perspectiva por lo que a una persona la verán de la misma importancia que los planos posteriores, es decir como si se tratara de un telón bidimensional.

Esta alteración perceptual se extiende a menudo a una incapacidad para reconocer y copiar formas y modelos; no es infrecuente encontrar niños de 8 ó 9 años de inteligencia normal incapaces de dibujar un rombo. En otras ocasiones encontramos la dificultad al tener que integrar un todo a partir de las partes aisladas. Es decir, les será difícil ensamblar figuras o reconocer un dibujo hecho con trazos cortados.

Aumentando el caos de este mundo perceptivo puede presentarse una marcada dificultad para reconocer posiciones: arriba-abajo, adelante-atrás $y$ derechaizquierda. Estas graves alteraciones per- 
ceptivas serán para el Paralítico Cerebral un escollo importante en la comprensión del mundo que lo rodea, sin la cual no podrá crear conceptos ni aprovechar verdaderamente su inteligencia.

Cada ser humano tiene un concepto de su propia imagen corporal que comienza a formarse a medida que explora el medio en que vive. El niño de pocos meses que agita los pies delante de sus ojos aún ignora que le pertenecen y luego en forma gradual va construyendo este concepto. Se piensa que normalmente relacionamos los objetos con nosotros mismos, como punto de referencia. Según esto, la formación de la imagen corporal sería muy importante en la construcción del mundo perceptivo, de por sí alterado en estos niños. Hay evidencia de que en el Paralítico Cerebral la imagen corporal se forma con dificultad y a menudo estos nin̄os creen que son los niños normales los que se comportan anormalmente. Presentan también frecuente distorsión en el dibujo de la figura humana, a la que trasladarian la imagen que tienen de sí mismos. Por otra parte, no distinguen en algunos casos, cuál es su derecha y su izquierda. A estos problemas se suma a veces una dominancia hemisférica no bien localizada, que conduce a problemas en la lectura, escritura y lenguaje.

Los puntos anteriormente tratados son en gran parte responsables de una serie de características del Paralítico Cerebral que configurarán una respuesta anormal a los estímulos. Es así como son niños con marcada distractibilidad es decir, tienen incapacidad de concentrar su atención por cierto tiempo en un objeto determinado, siendo por el contrario solicitados por todos los estimulos. Esta característica se puede observar en el niño normal, pero es patológica si se da con mayor intensidad y a edades más avanzadas que en éste.

Son incapaces de sintetizar, lo que en parte se deberia a conceptos confusos del mundo que los rodea, lo que a su vez repercute en incapacidad de elaborar pensamientos abstractos. Se piensa que en este punto también intervenga cierto grado de inmadurez emocional, pero ésto es dificil de comprobar.

Poseen además perseveración excesiva, es decir cuando logran fijar su atención en algo tienen dificultad en pasar a otro objeto. Esto se ve claramente en la apli- cación de test, porque cuando han dado una respuesta tienden a repetirla indefinidamente, y si por un momento logran concentrarse en un segundo tema, vuelven posteriormente al primero.

Se ha definido también en estos niños una aquiescencia excesiva que consiste en una reacción de igual intensidad a to. dos los estímulos con incapacidad de seleccionar objetos para atención especial, esta característica se ha atribuído a su dificultad en la diferenciación de figura $\mathrm{y}$ fondo.

En cuanto a la desinhibición se caracteriza por hiperactividad, inquietud, incapacidad de evitar reacciones motoras ante varios estímulos.

Como se ve, todas estas anomalias están estrechamente relacionadas entre si, dependiendo en gran parte de los defectos perceptivos anteriormente analizados y configurando una conducta aiterada.

Enfrentados al problema práctico de medir la inteligencia de estos niños, nos encontramos con que muchos de los tests ya estandarizados nos presentarán interrogantes acerca de su validez dados los defectos perceptivos y las características de los Paralíticos Cerebrales; sin embargo, nos servirán como una orientación acerca de las capacidades intelectuales de los pacientes. En toda evaluación debemos, además, considerar un cuestionario sobre lo que el niño es efectivamente capaz de hacer en la vida diaria, pero siempre comprobaremos, hasta donde sea posible, aquello que nos informen los padres, porque en su natural deseo de exaltar al niño pueden interpretar sus actividades como más inteligentes de lo que son en realidad.

Los tests intelectuales son pruebas arbitrarias que miden diferentes funciones de la inteligencia y que han sido estandarizados en grandes grupos de niños sanos. Es por esto que para nuestra evaluación elegiremos algunas pruebas compatibles con los trastornos motores de nuestros pacientes y seleccionadas de los tests de mayor validez. Para los niños de 1 a 4 años hemos elaborado una pauta basada en los tests de Gesell y Terman-Merrill, debiendo adecuarse algunos ítems para dar mayor facilidad a los coreoatetósicos especialmente. Así, en el reconocimiento de dibujos lineales y de escenas, los separamos y agrandamos con el fin de obtener una respuesta por medio de la direc- 
ción de la mirada en aquellos niños que carecen de lenguaje y de control de las manos y cuyo unico medio de expresión se aprovecha en esta forma.

A partir de los 5 años utilizamos las pruebas del Wechsler. Es este un tests que explora un número mayor de funciones intelectuales que otros tests, ya que considera pruebas de actuación junto a las verbales. Sin embargo, en los Paralíticos Cerebrales el resultado de estas pruebas son un índice relativo $\mathrm{y}$ sujeto a error de la inteligencia del nin̄o. Es así como los resultados de los ítems verbales pueden aparecer disminuídos en estos niños, porque su experiencia sensorial ha estado limitada por sus defectos motores que les han imposibilitado una vida normal y los han excluído de una enseñanza escolar e incluso familiar adecuadas.

Por otra parte las alteraciones perceptuales y de abstracción repercuten en los resultados de las pruebas de actuación aparentando un déficit intelectual que no siempre se corresponde con la realidad.

El Wechsler se compone de seis ítems verbales $\mathrm{y}$ seis de actuación, de los que se computan normalmente diez. Para los efectos de una valoración aproximada hemos seleccionado tres de los ítems verbales y tres de actuación del test de Wechsler que pasamos a describir brevemente, a fin de que se comprendan las dificultades $\mathrm{y}$ adaptaciones necesarias, a algunas de las cuales nos referiremos más adelante. La prueba de aritmética consiste en problemas de dificultad progresiva. La de similitudes consta de dos partes que se aplican de acuerdo a la edad del paciente; en la primera el niño debe completar una frase dada y en la segunda explicar verbalmente la semejanza entre dos cosas; de la riqueza de conceptos dependerá el puntaje que se le otorgue. En la prueba de repetición de nú. meros, el niño debe memorizar cifras de tres a nueve números en orden directo y de dos a ocho en orden inverso de acuerdo a su edad. En la completación de cuadros se exige descubrir lo que falta en un dibujo. La prueba de ensamblaje consiste en rompecabezas para armar en un tiempo limitado. Finalmente en el ítem de dibujo con cubos se pide imitar, con cubos de diferentes colores por los distintos lados, una figura modelo impresa en una tarjeta.

Como es sabido, estos niños se distraen fácilmente frente a estímulos secundarios ajenos a la prueba, es necesario, por lo tanto, un ambiente silencioso, sin colores 1lamativos y con el niño cómodamente instalado frente a una mesa de altura adecuada. Con frecuencia se requiere la presencia de la madre durante Ia aplicación del test ya que su ausencia suele desencadenar sentimientos de inseguridad que redundan en menor rendimiento. Exixsten diversos criterios frente a la aplicación del test, pero en general hay acuerdo en permitir ciertas concesiones por las dificultades motoras del niño, sin embargo nos parece conveniente encuadrarse dentro de un margen cercano a las condiciones estandarizadas a fin de obtener resultados valederos. Asi por ejemplo, en un coreoatetósico, en un nino con temblor intencional o en un cuadripléjico habrá que otorgar tiempo extra en las pruebas de actuación por su dificultad en los movimientos finos que exige la prueba, pero el examinador podrá percatarse cuando la solución ha sido captada dentro del tiempo permitido. En oposición a este caso está el del niño que con o sin dificultades manuales no es capaz de comprender el plan de ejecución, caso en el cual no debería prolongarse el tiempo de la prueba.

Otro defecto frecuente $y$ que dificulta Ia aplicación del test son los defectos graves del lenguaje que llegan a veces a la virtual carencia de éste o a la emisión de sonidos de difícil interpretación. En estos casos hay que guiarse por una sílaba indicadora y considerar que junto al problema intelectual por resolver, el niño tiene que luchar por expresar su idea, 1o que es particularmente notorio en la repetición de números, en que la cifra memorizada puede olvidarse por la demora en pronunciar cada número.

En los hemiplégicos y paraplégicos el déficit motor interfiere menos en la ejecución del test y éste se puede pasar en condiciones más cercanas a las estandarizadas.

Quisiéramos dejar el concepto de que una orientación acerca de la inteligencia del niño no es tan difícil como pudiera suponerse. La buena observación de su comportamiento durante el test a menu- 
do es mejor indice que el resultado numérico.

A fin de obtener una traducción práctica de la inteligencia de un niño se ha usado el concepto de "edad mental"; es decir indicar a qué edad corresponde la actuación de un niño dado. Al dividir la edad mental obtenida en un paciente por su edad cronológica tendremos el Cuo. ciente Intelectual o C.I.

A. Normal (los que poseen un C.I. de $90-100)$;

B) Subnormal educable (entre C.I. de 60 y 80 );

C) Deficiente profundo de difícil educación (C.I. $<$ de 60 ).

A pesar de esto conviene considerar que si vemos a un niño de 14 años con una capacidad mental de 7 podemos estimular a la familia para que le enseñe los pasos previos a la lectura y estritura, lo que podrá aprender a veces, tal como un niño normal de 7 años. En esto habrá que ser cautos y no forzar la enseñanza a fin de no levar a la ansiedad a un nino francamente incapaz.

Al realizar los tests encontraremos algunos pacientes de nivel intelectual normaI, ellos pueden asistir a escuelas corrientes; pero hay que considerar que un Paralítico Cerebral de inteligencia promedio puede tener considerable retraso escolar por sus dificultades visuomotoras.

Es papel del médico ayudar a que estos niños aprovechen al máximo sus posibilidades, dando algunas normas simples a los padres y maestros con el objeto de ayudarles a subsanar los defectos que ya conocemos. A continuación enumeraremos algunas medidas prácticas que faciliten el aprendizaje a través del mejoramiento de sus limitaciones psíquicas.

A. Trabajo y estudio fraccionados en períodos cortos y frecuentes, en medio de un ambiente con escasos estímulos sensoriales tal como lo requieren su fatigabilidad, su desinhibición motora, distractibilidad, y aquiescencia excesiva.

B. Dedicación individual dentro de las posibilidades del hogar o de la escuela. (En lo posible, clases con pocos alumnos $y$ de un nivel mental similar).

C. Formación de rutinas simples, sin cambios bruscos, que lo lleven a una mayor normalidad de su vida diaria; por Ej.: hábitos higiénicos, de vestimenta, estudio, etc.
D. Hay que procurar que la instrucción se desarrolle en base a trabajos de interés, que sean capaces de atraer su atención. A medida que esta capacidad se desarrolle podrá progresar en todos los campos. Además siempre se comenzará por tareas muy simples, en las que puedan triunfar, para después complicarlas en forma muy gradual $y$ de acuerdo al exito obtenido. Con este sistema lo estimularemos a la superación progresiva $y$ evitaremos las tensiones.

E. Ayudar al niño en etapas previas a la escolaridad. Es necesario insistir en el aprendizaje de colores, en la discriminación de formas y de tamaño. El niño normal aprende esto con escasa ayuda de Ios adultos. En Ios Paralíticos Cerebrales, por el contrario, deberá enseñarse la diferencia entre lo grande $y$ lo pequeño practicando sucesivamente con diferencias cada vez más finas. Adquieren con mayor facilidad el concepto de formas si se les enseña mediante juguetes sin colorido y en una etapa posterior pintados. La discriminación de formas es una etapa previa y fundamental del aprendizaje de las formas que constituyen el alfabeto. En una etapa posterior, por medio de juegos y rompecabezas, se le enseñará a reconocer y organizar formas simples lo que también contribuirá al aprendizaje de lectura y escritura.

Mediante estas normas y con un ambiente familiar adecuado lograremos el mayor desarrollo de la percepción, la concentración, el pensamíento abstracto, el discernimiento y la imaginación; elementos que le serán indispensables para su adaptación social y escolar.

Recordaremos por último que, siendo el aspecto externo de un niño con Parálisis Cerebral muy engañador, su evaluación intelectual es un esfuerzo que vale la pena realizar ya que sólo así conoceremos sus verdaderas posibilidades de éxito en los diversos aspectos de una terapia integral que abarque la rehabilitación motora $\mathrm{y}$ de lenguaje junto al aspecto pedagógico o vocacional.

Existen varios fármacos empleados con frecuencia a los que atribuye la virtud de aumentar la capacidad intelectual. Entre los de uso más dilatado está el Acido Glutámico. Sobre él hay numerosas experiencias con resultados muy dispares; la opinión de nuestro Servicio es que no aumenta la capacidad intelectual sino 
que, en el mejor de los casos, coloca al niño en un estado de mayor alerta mental que puede, a veces, ayudar a su mejor rendimiento.

En cuanto a los psicotónicos y timolépticos de reciente aparición: deanol, iproniazida, tofranil, etc. están aún en etapa de experimentación y no tenemos un concepto formado sobre su eficacia en el aspecto intelectual.

En resumen, por el momento no contamos con drogas de rendimiento comprobado en este campo, pero, ante padres muy angustiados convendrá usarlos como elementos psicoterápicos coadyuvante del resto del tratamiento.

\section{RESUMEN}

PERTURBACIONES DE LA INTELIGENCIA, SU EVALUACIÓN $X$ SU TRATAMIENTO EN LA PARÁLISIS CEREBRAL INFANTIL

El niño con parálisis cerebral tiene con frecuencia compromiso de la inteligencia y cuando existe puede ser de grados muy diversos; no hay correlación estricta entre el compromiso neurológico $\mathrm{y}$ el síquico.

Se analizan los defectos de la percepción y de la formación de la imagen corporal que condicionan en gran parte las características del conocimiento del $\mathrm{Pa}$ ralítico cerebral.

Se comenta el valor de los tests habituales aplicados a estos niños enumerándose algunos de los ítems utilizados y las adaptaciones necesarias en estos casos. A menudo la buena observación del niño durante el test es mejor índice que el resultado numérico, se recomienda además el uso de cuestionarios para investigar lo que el niño es efectivamente capaz de hacer en la vida diaria.

Finalmente se dan algunas medidas prácticas tendiente a subsanar los defectos $\mathrm{y}$ aprovechar la inteligencia de estos niños perturbados por Ios factores analizados.

\section{SUMMARY}

INTELLECTUAL DISTURBANCES, THEIR VALUATION AND THEIR TREATMENT IN THE INEANTILE CEREBRAL PALSY

The child with cerebral palsy frequently has an intellectual involment which may be of very different degrees; there is no strict correlation between the neurological and the psychic involvement.

Defects of the perception and those of the formation of the body conception which greatly condition the characteristics of the consciousness of the cerebrally palsied individual, are analyzed.

Value of the tests habitually applied to these children are commented and some of the items used and the adaptations necessary in these cases are enumerated. Often, the accurate observation of the child during the test is a better index than the numerical results; the use of questionaries is also recommended in the investigation of what the child is effectively able to do in the realities of life.

Finally, some practical measures which aim at obviating the defects and at making use of the intelligence of these children disturbed by the analyzed factors, are given.

\section{ZUSAMMENFASSUNG}

INTELLIGENZSTÖRUNGEN, IHRE BEWERTUNG UND IHRE BEHANDLUNG BEI DER ZEREBRALEN LAEHMUNG DES KINDES

Beim Kind mit zerebraler Lähmung ist häufig die Intelligenz mitbetroffen, und das kann in sehr verschiedenem Grade der Fall sein; es besteht keine starre Wechselbeziehung zwischen der neurologischen und der psychischen Schädigung.

Die Mängel der Perzeption und der Bildung der Körpervorstellung, die zum grossen Teil die Bewusstseinslage des zerebral Gelähmten bedingen, werden näher betrachtet.

Der Wert der üblichen, bei diesen Kindern angewandten Tests wird erörtert, und einige der gebrauchten Items und der in diesen Fällen nötigen Anpassungen werden aufgezählt. Meistens ist die genaue Beobachtung des Kindes während des Tests ein besserer Indikator als das zahlenmässige Resultat; empfehlenswert ist ausserdem die Benutzung von Fragebogen: zur Feststellung dessen, was das Kind tatsächlich im täglichen Leben $z u$ leisten vermag.

Endlich werden einige praktische Massnahmen angegeben, die darauf hinauslaufen, die Defekte zu beheben und die Intelligenz dieser durch die dargelegten Faktoren gestörten Kinder auszunutzen. 